\title{
$J$-NOETHERIAN INTEGRAL DOMAINS WITH 1 IN THE STABLE RANGE
}

WILLIAM J. HEINZER

Let $R$ be a commutative ring with identity. In $[1$, p. 29], Bass has shown that if the maximal spectrum of $R$ is noetherian of combinatorial dimension $d$ and if $R^{\prime}$ is a finite $R$-algebra, then $d+1$ defines a stable range for GL $\left(R^{\prime}\right)$. Estes and Ohm in [2] have considered some topics related to Bass's theorem mainly for the case when $R^{\prime}=R$. In particular, they prove, for any integer $n \geqq 0$, the existence of an integral domain $D$ such that the maximal spectrum of $D$ has dimension $n$ and 1 is in the stable range of $D$. They raise the question of whether there exists a domain $D$ with these properties for which the maximal spectrum of $D$ is noetherian. Our purpose is to give an affirmative answer to this question.

If $A$ is an ideal of $R$ then we let $J(A)$ denote the intersection of the collection of maximal ideals of $R$ containing $A$ and $J=\{$ ideals $A$ of $R \mid J(A)=A\}$. We say that $R$ is $J$-noetherian if the ideals of $J$ satisfy the ascending chain condition. This is equivalent to the statement that the maximal spectrum of $R$ is noetherian. The dimension of the maximal spectrum of $R$ is $n$ provided there is a chain $P_{0}<P_{1}$ $<\cdots<P_{n}$ of prime ideals of $R$ which are in the set $J$ but no chain of longer length. In this case, we say that $R$ has $J$-dimension $n$. A positive integer $t$ is said to be in the stable range of $R$ if for $s \geqq t$ whenever $a_{1}, a_{2}, \cdots, a_{s+1}$ are elements of $R$ such that $\left(a_{1}, a_{2}, \cdots, a_{s+1}\right)=R$, then there exist $b_{1}, \cdots, b_{s} \in R$ such that $\left(a_{1}, a_{2}, \cdots, a_{s+1}\right)$ $=\left(a_{1}+b_{1} a_{s+1}, \cdots, a_{s}+b_{s} a_{s+1}\right)$. We can now state our result as follows.

THEOREM. For each positive integer $n$ there is a J-noetherian domain $D$ which has 1 in the stable range and has $J$-dimension $n$.

Our examples are obtained by using an existence theorem due to Jaffard [3, p. 78]. Jaffard has shown that if $G$ is a lattice-ordered abelian group then there is an integral domain $D$ which has $G$ as its group of divisibility. His construction is carried out by using the group ring $B(G)$ of $G$ with respect to an arbitrary field $F$. The elements of $B(G)$ may be regarded as formal sums $\sum_{i=1}^{n} a_{i} X^{\boldsymbol{o}_{i}}$ where $a_{i} \in F$ and $g_{i} \in G$. We wish now to observe that Jaffard's construction

Received by the editors August 10, 1967. 
gives, in fact, a Bezout ${ }^{1}$ domain $D$ which has 1 in the stable range. ${ }^{2}$ The domain $D$ is obtained by defining a map $\phi$ from $B(G)^{*}$, the nonzero elements of the domain $B(G)$, into $G$. We can write an element of $B(G)^{*}$ uniquely in the form $\sum_{i=1}^{n} a_{i} X^{v_{i}}$ where the $g_{i}$ are distinct elements of $G$ and the $a_{i}$ are nonzero elements of the field $F$. Then $\phi\left(\sum_{i=1}^{n} a_{i} X^{y_{i}}\right)=\inf \left\{g_{i}\right\}$. We extend $\phi$ to $E^{*}$, the nonzero elements of the quotient field of $B(G)$, by defining $\phi(p / q)=\phi(p)-\phi(q)$ for $p$, $q \in B(G)^{*}$. If $D^{*}=\left\{y \in E^{*} \mid \phi(y) \geqq 0\right\}$ then $D=D^{*} \cup\{0\}$. To show that 1 is in the stable range of $D$ and that $D$ is Bezout we show that for $p, q \in E^{*}$ there is an element $r$ of $D$ such that $p D+q D=(p+r q) D$. Since $\phi(p)=\phi\left(X^{\phi(p)}\right)$ we have $p=X^{\phi(p)} u_{1}$ where $u_{1}$ is a unit of $D$. Similarly $q=X^{\phi(q)} u_{2}$ where $u_{2}$ is a unit of $D$. If $\phi(p) \leqq \phi(q)$, then we choose $r=0$. Since $\phi(q / p)=\phi(q)-\phi(p) \geqq 0$ we have $q \in p D$ and hence $p D+q D=p D$. If $\phi(p) \$ \phi(q)$ then we set $r=u_{1} / u_{2}$. Since $u_{1}$ and $u_{2}$ are units of $D, \phi(r)=\phi\left(u_{1}\right)-\phi\left(u_{2}\right)=0$ and $r \in D$. We have $p+r q$ $=u_{1} X^{\phi(p)}+u_{1} X^{\phi(q)}=u_{1}\left(X^{\phi(p)}+X^{\phi(q)}\right)$. And because $\phi(p) \neq \phi(q)$ we have $\phi(p+r q)=\phi\left(u_{1}\right)+\phi\left(X^{\phi(p)}+X^{\phi(q)}\right)=0+\inf \{\phi(p), \phi(q)\}$. Hence $\phi(p /(p+r q))$ and $\phi(q /(p+r q))$ are positive and $p D+q D=(p+r q) D$. It follows that $D$ is a Bezout domain. Moreover, if $\left(a_{1}, a_{2}, \cdots, a_{s+1}\right)=D$, then there exist elements $b_{i}$ of $D$ such that $\left(a_{i}, a_{s+1}\right)=\left(a_{i}+b_{i} a_{s+1}\right)$ for $i=1, \cdots, s$. Hence $\left(a_{1}, \cdots, a_{s+1}\right)=\left(a_{1}+b_{1} a_{s+1}, \cdots, a_{s}+b_{s} a_{s+1}\right)$ and 1 is in the stable range of $D$.

Let $G^{+}$denote the set of positive elements of $G$. By an ideal of $G$ we will mean a nonempty subset $I$ of $G^{+}$with the following properties: (1) $0 \notin I$, (2) if $a \in I$ and $b>a$, then $b \in I$, (3) if $a, b \in I$ then $\inf \{a, b\} \in I$. If, in addition, the complement of $I$ in $G^{+}$is closed under addition then we say that $I$ is a prime ideal. With each ideal $I$ of $G$ we associate the subset $\phi^{-1}(I) \cup\{0\}$ of $D$. It is straightforward to check that this gives a one-to-one inclusion preserving correspondence between the ideals of $G$ and the proper integral ideals of the Bezout domain $D$. Moreover, $I$ is a prime ideal of $G$ if and only if $\phi^{-1}(I) \cup\{0\}$ is a prime ideal of $D$.

We proceed to construct a lattice-ordered abelian group $G$ which has the following properties:

1. There is a chain $I_{0}<I_{1}<\cdots<I_{n}$ of prime ideals of $G$ but no chain of longer length.

2. Every prime ideal of $G$ is an intersection of maximal ideals.

3. For each ideal $I$ of $G$ there are only finitely many prime ideals of $G$ which are minimal with respect to the property of containing $I$.

It follows from our previous observations that a domain $D$ con-

1 We say that $D$ is a Bezout domain if every finitely generated ideal of $D$ is principal.

${ }^{2}$ For the proof of this fact and for several other helpful suggestions concerning this paper, I wish to thank Jack Ohm. 
structed by means of Jaffard's theorem which has $G$ as its group of divisibility will have Krull dimension $n+1$, each proper prime ideal of $D$ will be an intersection of maximal ideals, and each ideal of $D$ will have only finitely many minimal prime divisors. Since $D$ is finite dimensional this last property implies that $D$ is $J$-noetherian [2]. Moreover, $D$ will have $J$-dimension either $n$ or $n+1$. If $G$ has infinitely many minimal primes then (0) is an intersection of maximal ideals of $D$ and $\operatorname{dim}_{J} D=n+1$; otherwise $\operatorname{dim}_{J} D=n$.

We will say that a group $G$ which satisfies property 1 above has dimension $n$.

Our construction of a group $G$ which has properties 1,2 , and 3 is based on the following two lemmas.

LEMMA 1. Let $\left\{H_{\alpha}\right\}$ be a family of lattice-ordered abelian groups and let $H$ be the weak direct sum of the $H_{\alpha}$ 's, where $H$ is ordered by defining $\left\{a_{\alpha}\right\} \geqq\left\{b_{\alpha}\right\}$ if and only if $a_{\alpha} \geqq b_{\alpha}$ for each $\alpha$. Let $p_{\alpha}: H \rightarrow H_{\alpha}$ be the canonical projection homomorphism. Then each prime ideal $Q$ of $H$ is of the form $p_{\alpha}^{-1}\left(Q_{\alpha}\right) \cap H^{+}$where $Q_{\alpha}$ is a prime ideal of some $H_{\alpha}$ and $H^{+}$ is the set of positive elements of $H$.

Using properties of the weak direct sum and the definition of a prime ideal in $H$ the proof of Lemma 1 is straightforward and will be omitted.

It follows from Lemma 1 and the fact that elements in the weak direct sum have only finitely many nonzero coordinates that if $\left\{H_{\alpha}\right\}$ is a collection of lattice-ordered abelian groups such that each $H_{\alpha}$ satisfies properties 1,2 and 3 above, then $H=\sum_{\alpha} H_{\alpha}$ also satisfies these properties. Moreover, if there are infinitely many $\alpha$ then $H$ has infinitely many minimal primes.

Lemma 2. Suppose that $H$ is a lattice-ordered abelian group which satisfies properties 1, 2, and 3 stated above. Assume in addition that $H$ has infinitely many minimal primes. Let $T$ be a totally ordered archimedean group and let $K=T \oplus H$ where we order $K$ by defining $(a, h)$ $\geqq\left(a^{\prime}, h^{\prime}\right)$ if and only if $a>a^{\prime}$ or $a=a^{\prime}$ and $h \geqq h^{\prime}$. Then $K$ is a latticeordered abelian group having dimension $n+1$ and $K$ satisfies properties 2 and 3 stated above.

Proof. We note first that $Q=\{(a, h) \in K \mid a>0\}$ is the unique minimal prime ideal of $K$ and that every ideal of $K$ compares with $Q$. It is clear that $Q$ is a prime ideal; and that $Q$ is minimal follows from the fact that if $a, a^{\prime} \in T$ with $a>0$, then for some positive integer $n$, $n a>a^{\prime}$. If $(a, h) \in K^{+}-Q$ then $a=0$ so $(a, h)<q$ for any $q \in Q$. Hence any ideal containing $(a, h)$ contains $Q$, and it follows that every ideal of $K$ compares with $Q$. 
If $I$ is an ideal of $K$ which properly contains $Q$ then let $I_{H}$ $=\{h \in H \mid(0, h) \in I\} . I_{H}$ is an ideal of $H$ and $I$ is a prime ideal of $K$ if and only if $I_{H}$ is a prime ideal of $H$. The mapping which associates $I$ with $I_{H}$ is a one-to-one inclusion preserving correspondence between the ideals of $K$ which properly contain $Q$ and the ideals of $H$. Since $I$ is prime if and only if $I_{H}$ is prime, we conclude that each nonminimal prime of $K$ is an intersection of maximal ideals and that the ideals of $K$ satisfy property 3 . Finally, the fact that $H$ has infinitely many minimal primes implies that $Q$ is the intersection of the primes of $K$ which properly contain $Q$. Hence $K$ also satisfies property 2 . This completes the proof of Lemma 2.

By starting with a totally ordered archimedean group and applying Lemmas 1 and 2 we can now obtain for any positive integer $n$ a lattice-ordered abelian group $G_{n}$ which satisfies properties 1,2 and 3 and has a unique minimal prime. The domain $D_{n}$ constructed by means of Jaffard's theorem which has $G_{n}$ for its group of divisibility is $J$-noetherian, has 1 in the stable range, and has $J$-dimension $n$. Moreover, the fact that $D_{n}$ is a Bezout domain and hence is Prüfer implies that $D_{n}$ has the same prime ideal structure as any Kronecker function ring $D_{n}^{*}$ of $D_{n}$ [4]. In fact each ideal of $D_{n}^{*}$ is the extension of an ideal of $D_{n}$ and each ideal of $D_{n}$ is the contraction of its extension. It follows that $D_{n}^{*}$ is also $J$-noetherian and has $J$-dimension $n$. This gives an affirmative answer to the question raised in [2] concerning the existence of $J$-noetherian Kronecker function rings of arbitrary $J$-dimension.

\section{REFERENCES}

1. H. Bass, K-theory and stable algebra, Inst. Hautes Etudes Sci. Publ. Math. No. 22 (1964), 5-60.

2. D. Estes and J. Ohm, Stable range in commutative rings, J. Algebra 7 (1967), 343-362.

3. P. Jaffard, Les systèmes d'idéaux, Dunod, Paris, 1960.

4. W. Krull, Beiträge zur Arithmetik kommutativer Integritätsbereiche. I, Math Z. 41 (1936), 544-577.

Louisiana State. University 